\title{
High precision subsurface photodisruption in human sclera
}

Zachary S. Sacks

University of Michigan

Center for Ultrafast Science

Ann Arbor, Michigan 48109-2099

\author{
Ron M. Kurtz \\ University of Michigan \\ Center for Ultrafast Science \\ Ann Arbor, Michigan 48109-2099 \\ and \\ University of Michigan \\ Kellogg Eye Center \\ Department of Ophthalmology \\ Ann Arbor, Michigan 48105 \\ and \\ University of California \\ Department of Ophthalmology \\ Irvine, California 92697
}

\section{Tibor Juhasz}

University of Michigan

Center for Ultrafast Science

Ann Arbor, Michigan 48109-2099

and

University of Michigan

Kellogg Eye Center

Department of Ophthalmology

Ann Arbor, Michigan 48105

and

University of Michigan

Biomedical Engineering

Ann Arbor, Michigan 48109

\section{Gerard A. Mourau}

University of Michigan

Center for Ultrafast Science

Ann Arbor, Michigan 48109-2099

\begin{abstract}
Background and objectives: Femtosecond pulses can generate high precision subsurface photodisruption in transparent tissues, such as the cornea. We used femtosecond laser technology to demonstrate early proof of concept for high precision subsurface photodisruption in the translucent sclera. This technique may ultimately enable novel surgical procedures for the treatment of glaucoma and/or presbyopia. Study design/materials and methods: Microjoule femtosecond pulses from two different sources, 1060 and $775 \mathrm{~nm}$, were used to make subsurface incisions in human sclera in vitro. Scleral tissue was dehydrated to improve translucency at these wavelengths. The beam was focused to a $1.5(775 \mathrm{~nm})$ or $5 \mu \mathrm{m}$ spot size $(1060 \mathrm{~nm})$ and scanned below the tissue surface at various depths to produce four incision patterns. Results: Photodisruption on the backsurface of the sclera was achieved without damage to overlying tissue. Several types of intrascleral incisions were made, including transcleral channels and grooves for scleral implants. Conclusions: High precision, subsurface scleral photodisruption can be achieved in vitro for a variety of intrascleral incisions. Further studies are required to determine if this technique is applicable in vivo for actual surgical applications. (c) 2002 Society of Photo-Optical Instrumentation Engineers. [DOI: $10.1117 / 1.1482381]$
\end{abstract}

Keywords: femtosecond pulse; glaucoma; presbyopic scleral implant; transparency inducing.

Paper JBO 20051 received Nov. 14, 2000; revised manuscript received Jan. 24, 2002; accepted for publication Feb. 1, 2002.

\section{Introduction}

Photodisruption occurs when laser power intensity is large enough to induce optical breakdown in a material: the laser pulse transforms the material into plasma. The strong optical intensities required for photodisruption can be achieved at the focus of a high peak power laser beam. Breakdown occurs only where the intensity is larger than the threshold level. Below this threshold level, the laser pulse passes through the material without causing damage. Thus, the location of optical breakdown can be controlled to occur only at the focus of the beam where the intensity exceeds the threshold level. If the laser is focused beneath the surface of a material, subsurface breakdown occurs only at the focus. No damage occurs in the material that the beam was focused through. Complete subsurface incisions of any shape are possible by scanning the focal spot beneath the surface.

Photodisruption is an intensity dependent process. Therefore, pulse energy, which is associated with collateral damage processes, can be decreased by decreasing the pulse duration.

Address all correspondence to Ron M. Kurtz, University of California, Irvine 118 Med Surge I, Irvine, CA 92697. Tel: 949-824-6256; Fax: 949-824-4015; E-mail: rkurtz@uci.edu
If femtosecond pulses are used, the pulse energy can be decreased (microjoule level or lower) so that most of the pulse passes through the material with only a small fraction being absorbed. ${ }^{1}$ When photodisruption occurs near the threshold energy level, most of the absorbed energy is used to create the plasma with only a small amount of the absorbed energy dissipated by other mechanisms, such as shock wave formation, bubble oscillation, and thermal diffusion. ${ }^{2-4}$ Femtosecond pulses, therefore, allow precise subsurface disruption with almost no collateral damage. ${ }^{1,5}$ The diameter of the disrupted volume can be on the order of microns, allowing computer controlled, scanning delivery systems to create almost any subsurface incisional patterns. ${ }^{2}$

Photodisruption using femtosecond pulses has been used to create high precision subsurface incisions within the transparent cornea, without damaging overlying or surrounding tissues. ${ }^{1}$ In contrast to corneal tissue, the sclera scatters visible light, spreading the pulse in both space and time, ${ }^{6}$ making it difficult to maintain the tight focus and short pulse duration needed for well-confined photodisruption. The ability to focus

1083-3668/2002/\$15.00 ㄷ 2002 SPIE 




(a)

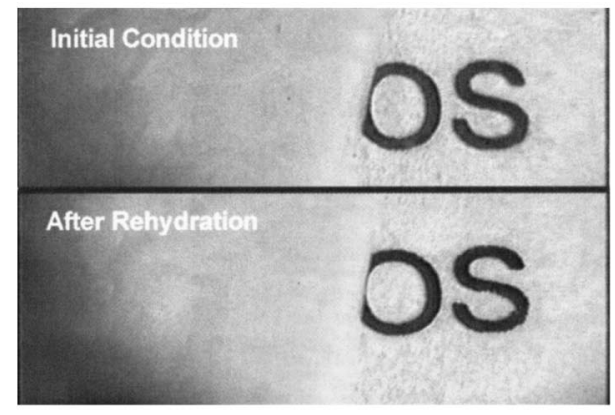

(b)

Fig. 1 Inducing transparency in human sclera using Hypaque. (a) Hypaque was used to induce transparency in a full thickness (0.5 mm) piece of human sclera in about 20 min. (b) The process is reversible if the Hypaque is removed and saline solution is added.

light through sclera is limited, especially at wavelengths less than $800 \mathrm{~nm}$. At longer wavelengths, where scattering is reduced, ${ }^{6}$ water in the tissue absorbs laser energy, thereby making the intensity levels necessary for photodisruption harder to achieve. Dehydrating agents, such as Hypaque $76^{7}$ have been shown to increase scleral transparency by reducing scattering, ${ }^{8}$ without damaging the tissue if used for short periods of time. ${ }^{9}$ The mechanism of action of this agent can be understood based on the fiber structure of the sclera.

Human sclera is composed of collagen fibrils embedded in a host matrix material. Sclera appears white because of the size (30-300 $\mathrm{nm}$ diameter), packing ( $285 \mathrm{~nm}$ average period), and the index mismatch between the fibers $(n=1.474)$ and the host matrix $(n=1.345) .{ }^{10}$ Bragg scattering for sclera occurs at wavelengths shorter than $800 \mathrm{~nm}$, assuming an index of 1.4 for sclera, ${ }^{8}$ thus making sclera an effective diffuser for visible light. Sclera can be made clear by decreasing the index mismatch between the fibers and the host material matrix through dehydration. The index of the background material is raised as the water $(n=1.33)$ is removed. Several dehydrating agents have been examined previously, including Trazograph and glucose. ${ }^{8}$

Figure 1 shows an example of transparency induced in in vitro human sclera. A tissue that is normally white can be made transparent enough to read through upon dehydration. We hypothesized that if such dehydrating agents cause significant reduction in scattering, then femtosecond pulses of normally scattered wavelengths could be focused strongly beneath the surface to permit subsurface photodisruption, enabling development of a number of potential scleral procedures.

In the first section of this paper, qualitative measurements of laser beam spot sizes through sclera are described to show the need for inducing transparency if subsurface photodisruption is to be achieved at 775 and $1060 \mathrm{~nm}$. In the second part, some examples of surface incisions are shown in human sclera. These incisions are completely subsurface and a variety of shapes with almost no collateral damage.

\section{Focusing Through Hydrated and Dehydrated Sclera}

Tissue from human globes, not suitable for transplantation, was obtained from the Midwest Eye Bank and Transplantation
Center one to four weeks after harvest. Full thickness scleral pieces (approximately $1 \mathrm{~cm}$ square and approximately $0.5 \mathrm{~mm}$ thick) were cut from the limbal region. Samples were preserved for up to several months in $95 \%$ ethanol solution. ${ }^{11}$ Prior to experimentation, samples were soaked in physiological saline solution for several hours. Some samples were then dehydrated in Hypaque 76 until becoming maximally transparent, usually within $15 \mathrm{~min}$.

The transmission spectrum of the tissue was measured in a spectrophotometer. The spectra obtained were forward scattered spectra - the light scattered in a small cone angle around the incident beam-as no integrating spheres were used. ${ }^{12}$ The forward scattered light is believed to be an indicator of the light that is focusable through the sclera. As shown in Figure 2, transmission of hydrated sclera is never greater than $10 \%$, peaking at $1700 \mathrm{~nm}$. Upon dehydration, the transmission greatly increases across the entire spectrum, especially for wavelengths in the near infrared. The transmission is mainly controlled by scattering in the visible spectrum be-

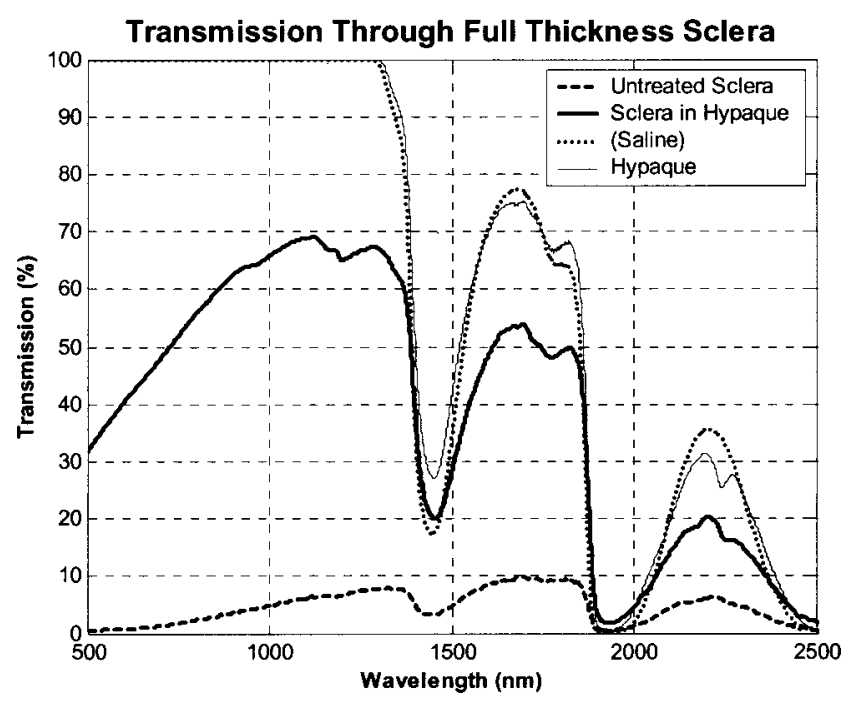

Fig. 2 Transmission spectra of hydrated and dehydrated sclera along with saline and Hypaque. All samples were approximately $0.5 \mathrm{~mm}$ thick. Hydrated sclera is an effective scatter for visible light since the transmission never goes above $10 \%$. Dehydrating the sclera with $\mathrm{Hy}$ paque greatly increases the transmission over the entire range. 


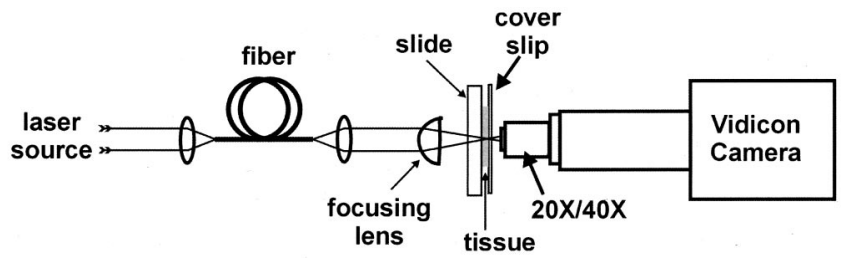

Fig. 3 Spot size measurement setup. A clean spatial mode created by a single mode optical fiber was focused through the sclera mounted between a microscope slide and a cover slip. The backsurface of the tissue was imaged to a vidicon with a $20 \times$ or $40 \times$ microscope objective.

cause of absence of absorbing chromophores in this region in sclera. Attenuation occurs at longer wavelengths due to the absorption spectrum of water, most notably seen by the strong peaks at 1450 and $1900 \mathrm{~nm}$. Transmission at 775 and $1060 \mathrm{~nm}$ is above $60 \%$. The difference in transmission is not expected to affect the results of photodisruption since photodisruption depends on the intensity of the pulse and not the linear absorption.

Since scattering is the dominant mechanism controlling transmission, transmission spectra of sclera are not good indicators of the focusability of a laser beam beneath the surface. The next experiment was performed to qualitatively describe the size of the focal spot on the backsurface of sclera. This study was performed only qualitatively since: (1) inhomogeneities in the sclera change the spot size from location to location, (2) the technology of the camera used is not suited to
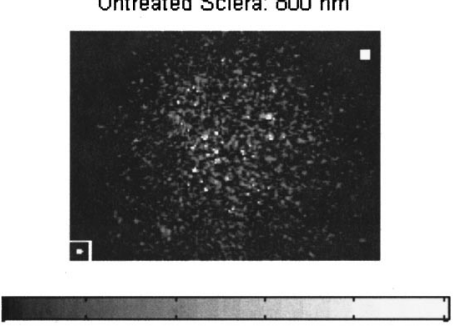

take exact measurements due to dynamic nonuniformity and gain curves, and (3) the exact spot size obtained in photodisruption will be altered by nonlinear processes, such as selffocusing. However, the spot images obtained are enough to explain the observed physical processes.

The spot size measurement setup is shown in Figure 3. Since different lasers were used, the profiles were approximately made to be the same by passing the beam through a single mode step index fiber to obtain a $L P_{01}$ (near Gaussian) spatial mode. The intensity of the beam was attenuated to be low enough to prevent nonlinearities in the fiber. The beam was then focused using a 0.5 numerical aperture (NA) aspheric lens (Thorlabs C240TM) to the backsurface of a piece of sclera sandwiched between a microscope slide and cover slip. The backsurface of the sclera was imaged using a $20 \times$ microscope objective to an Electrophysics 7290A-06 vidicon camera. (This camera was chosen to examine the spot sizes at wavelengths up to $2200 \mathrm{~nm}$.) ${ }^{13}$

Before the tissue was examined, the size of the unscattered beam was measured by using an equivalent slab of water $(0.5$ $\mathrm{mm}$ ) between the glass slides used to mount the tissue. Figure 4(a) shows the minimum spots obtained for 775 and $1060 \mathrm{~nm}$ after focusing through a full thickness $(0.5 \mathrm{~mm})$ piece of tissue. The square in the top right hand corner is $10 \mu \mathrm{m}$ on edge. In the bottom left corner is the beam after passing through an equivalent thickness of saline. The unscattered beam is smaller than $10 \mu \mathrm{m}$ in diameter. The large size is due to underfilling the focusing lens with the incident beam and ab-

(a)


(b)

Fig. 4 Minimum spot sizes of a focused beam through human sclera. The bottom left corner shows the smallest spot obtained by focusing through an equivalent thickness $0.5 \mathrm{~mm}$ slab of water. A square $10 \mu \mathrm{m}$ on edge is shown in the top right corner. The bar beneath each picture is the color map: left is not light (black), right is maximum light intensity (white). (a) In normal hydrated tissue, focusing at 775 and $1060 \mathrm{~nm}$ is not possible because of scattering. Image taken with $20 \times$ magnification. (b) When water is removed from the tissue using a dehydrating agent, the tissue becomes clear as the index is matched between the collagen fibers and host matrix. The smallest focusable spot through sclera is about the same size as the beam focused through water. Image taken with $40 \times$ magnification. 


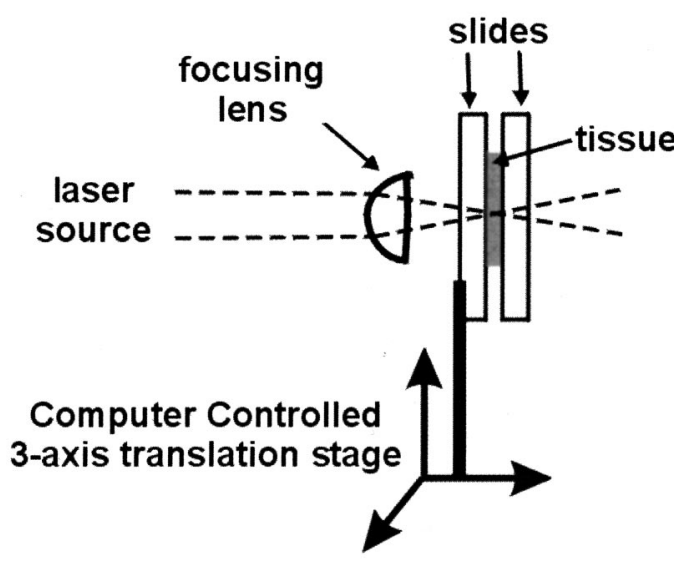

Fig. 5 Schematic of photodisruptive cutting experiment. A computer controlled translation stage was used to move the tissue sample, sandwiched between two microscope slides, in the focus of a femtosecond laser beam.

errations due to focusing through the material. As predicted at $775 \mathrm{~nm}$, the size of the intensity distribution emerging from the sclera did not change on the position of the focusing lens since scattering is very strong. The emergent beam is many times larger than the unscattered spot and full of speckle. At $1060 \mathrm{~nm}$, the distribution is smaller, but still heavily scattered. These images clearly show that focusing to the backsurface of the tissue is not possible using these wavelengths. Hence, backsurface photodisruption is not possible using these wavelengths, which has also been our experience: breakdown occurs at the front surface of the tissue since the breakdown threshold is reached there first as the pulse energy is increased. Figure 4(b) shows the minimum spot sizes obtained after treating the tissue with Hypaque 76. The spot size decreases to almost that of the unscattered beam. The strong focusing of the beam should permit controlled backsurface photodisruption.

\section{Subsurface Photodisruption in Sclera Using a Dehydrating Agent: Methods}

The scleral tissue sample (typically $0.5 \mathrm{~mm}$ thick) was placed between two microscope slides (each $1 \mathrm{~mm}$ thick) and mounted on a computer controlled translation stage in order to move the tissue with respect to the laser focus as shown in Figure 5. All incisions were made by single-shot laser exposure: the sample was translated fast enough so that each piece of treated tissue was hit only once by the laser. Typical translation speeds were $5 \mathrm{~mm} / \mathrm{s}$, which yields $5 \mu \mathrm{m}$ laser spot spacing for a $1 \mathrm{kHz}$ pulsed laser system. Different surgical patterns were formed by scanning the spot in a raster pattern to achieve the desired incision. The beam's initial position was determined using the visible light produced by photodisruption of glass.

Femtosecond lasers were used to demonstrate the possibility of achieving subsurface photodisruption. Two different lasers were used because of the availability issues. (This paper is not meant to be a comparison between the two lasers.) The first laser had typical operating parameters of $5 \mu \mathrm{J}, 1 \mathrm{kHz}$, $500 \mathrm{fs}$, and $1.06 \mu \mathrm{m},{ }^{14}$ and the second at $5 \mu \mathrm{J}, 1 \mathrm{kHz}, 150 \mathrm{fs}$, and $775 \mathrm{~nm}$. For both lasers, the beam was focused through a 0.5 NA aspheric lens (ThorLabs C240TM) corrected for wave front flatness. The spot size in air was measured to be $5 \mu \mathrm{m}$ full width half maximum (FWHM) at the focus of the lens for the $1060 \mathrm{~nm}$ light and $1.5 \mu \mathrm{m}$ for the $775 \mathrm{~nm}$ light. No compensation was made for potential aberrations produced by the microscope slides and dehydrated sclera. ${ }^{15,16}$ Tissue inhomogeneities inherent in the tissue and nonlinear self-focusing

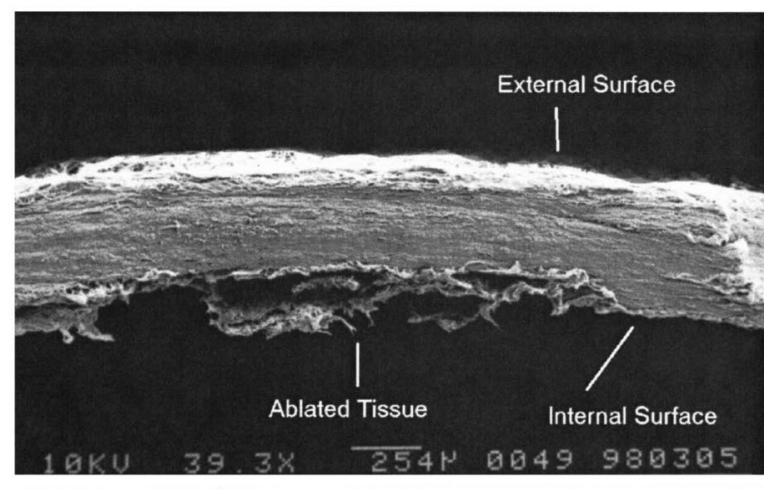

(a)

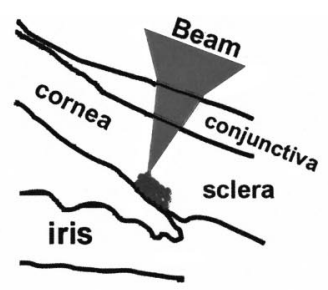

(b)



(c)

Fig. 6 SEM of back surface partial thickness channel that may be useful for a trabeculectomy. Debris was removed using a weakly pressurized saline stream. (a) Incision shown in cross section. Note no collateral tissue damage is present above the incision. (Laser parameters: $8 \mu$ ), 500 fs, $1060 \mathrm{~nm}, 1 \mathrm{kHz}, 5 \mu \mathrm{m}$ FWHM spot, $10 \mu \mathrm{m}$ spot separation.) (b) Schematic of a subsurface laser trabeculectomy. (c) Raster pattern of the laser focus. The gray plane shows the dissection cross-section in (a). 




(a)

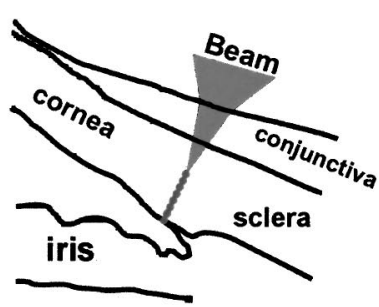

(b)



(c)

Fig. 7 Femtosecond laser created transscleral channel. (a) A channel with a minimum width was demonstrated. The channel width is estimated at $10 \mu \mathrm{m}$ or less from this cross section. A plane was scanned in order to find the channel upon dissection. (Laser parameters: $4.2 \mu \mathrm{J}, 150 \mathrm{fs}, 775 \mathrm{~nm}$, $1 \mathrm{kHz}, 2 \mu \mathrm{m}$ FWHM spot, $1.5 \mu \mathrm{m}$ spot separation.) The minimum width channel was made through human sclera. (b) Potential application of creating a drainage passageway. (c) Scan pattern.

also make it difficult to determine the spot size in the tissue. The goal here was simply to produce a surgical effect in a normally scattering tissue.

Treated scleral samples were fixed in $2 \%$ glutaraldehyde and prepared for scanning electron microscopy (SEM) immediately after laser exposure. Since this process includes dehy- dration, distortion secondary to shrinkage and gross movement of structures is possible. ${ }^{17,18}$

\section{Subsurface Photodisruption Results}

In all of the examples that follow, three pictures are shown in the figures. Part (a) shows the electron micrograph of the
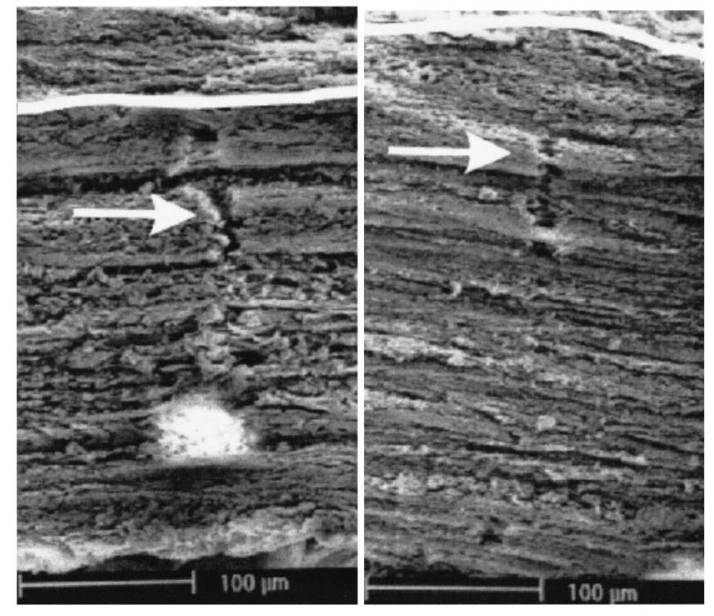

(a)

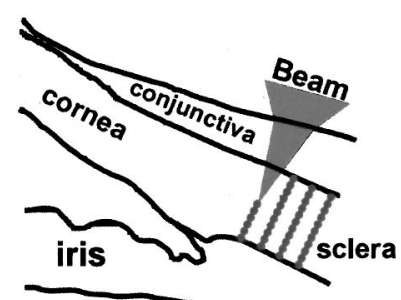

(b)

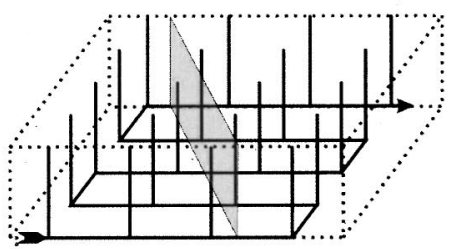

(c)

Fig. 8 Cross section of channel created in human sclera. A channel the width of the beam was created in the sclera using $1.0 \mu \mathrm{m}$ spot separation in the vertical direction (perpendicular to the tissue surface). The channel penetrates the full scleral thickness as verified in top and bottom SEM examination. (a) The channels are not seen to penetrate the entire surface here because the sectioning cut intersects only a portion of the channel. (Laser parameters: $5 \mu$ J, 500 fs, $1060 \mathrm{~nm}, 1 \mathrm{kHz}, 5 \mu \mathrm{m}$ FWHM spot, $0.1 \mu \mathrm{m}$ vertical spot separation.) (b) Such channels may be useful for altering the hydraulic conductivity of sclera for increased fluid outflow. (c) Raster pattern for the laser focus. 


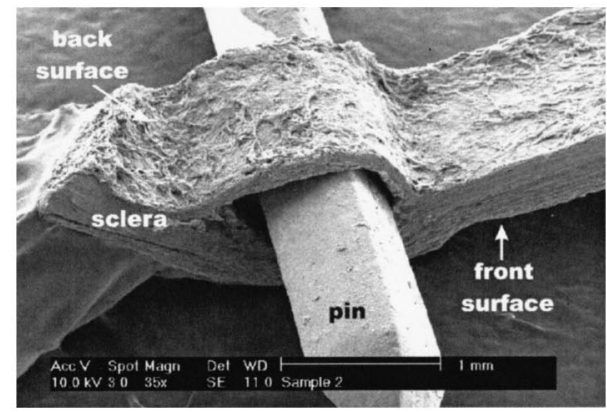

(a)

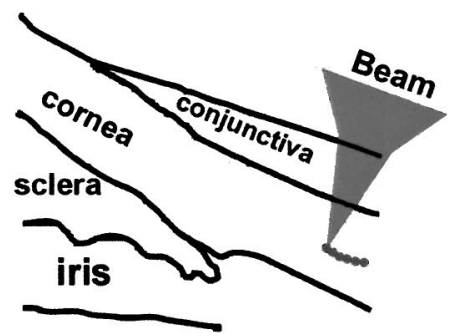

(c)

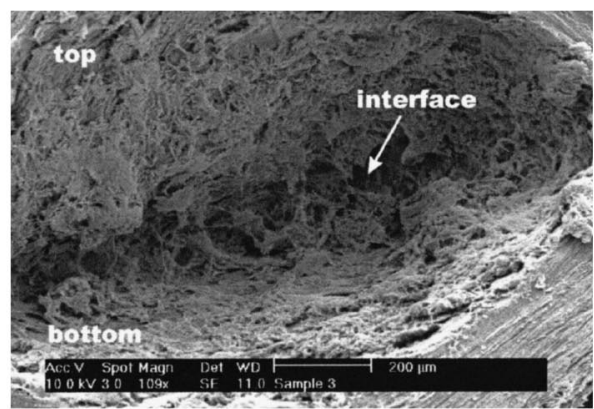

(b)

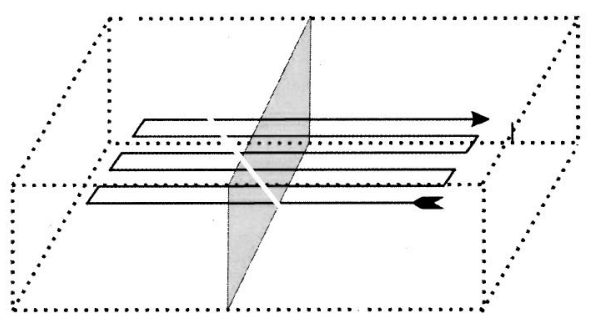

(d)

Fig. 9 Channel for presbyopia implants. (a) The pocket was easily opened with forceps and a pin was placed inside. (b) Close up of the incised interface. (Laser parameters: $6 \mu$ J, $150 \mathrm{fs}, 775 \mathrm{~nm}, 1 \mathrm{kHz}, 1.5 \mu \mathrm{m}$ FWHM spot, $5 \mu \mathrm{m}$ spot separation.) (c) A subscleral pocket is created $3 \mathrm{~mm}$ posterior to the limbus. (d) Raster pattern for the pocket.

treated tissue. Part (b) shows the treatment geometry in a full globe. Part (c) shows the scanning pattern of the laser spot.

\subsection{Partial Thickness Channel Creation}

Figure 6 shows the backsurface ablation created by focusing the femtosecond laser pulses through the tissue. Note that no damage occurred above (superficial to) the ablated region, even though the laser beam was focused through this portion of the tissue. There is no technical limit to create deeper channels, since the beam is moved from the inside of the tissue outwards. This incision pattern could be used to perform transscleral procedure analogous to deep sclerectomy, where a block of inner surface sclera is removed with minimal disruption to overlying layers.

\subsection{Full Thickness Channel Creation}

Figure 7 demonstrates the narrowest transscleral channel possible using our experimental setup. The beam was focused through the tissue to the inner surface and then advanced outward (towards the focusing lens). A plane was cut by the laser in order to find the incision after sectioning. The channel has an estimated width of $10 \mu \mathrm{m}$, which is probably even narrower but is obscured by the electron micrograph processing. This incision demonstrates the ability to cut a full thickness fistula that may be useful for draining aqueous for the treatment of glaucoma. ${ }^{19}$ Partial thickness cuts may be useful in altering the mechanical properties of the sclera for the treatment of presbyopia. ${ }^{20}$

\subsection{Creation of Tissue Pores}

A grid of full-thickness pores were created using the pattern shown in Figure 8(b). This treatment may be useful in changing the bulk properties of the tissue, including the tissue's hydraulic conductivity. Two representative channels were identified after sectioning, each approximately $10 \mu \mathrm{m}$ wide. Penetration of the laser beam to the superficial and deep surfaces was indicated by a regular array of photodisrupted tissue at the channel openings at both surfaces (not shown).

\subsection{Scleral Pocket Creation}

Intrascleral pocket incisions were successfully created [Figure 9(c)], using the pattern schematized in Figure 9(a). A $5 \mu \mathrm{m}$ spot separation was used to first create the pocket, which was then opened manually with a forceps. A metal pin was placed into the pocket to demonstrate the complete opening, while the surface quality of the separated tissue plane at both the superficial and deep portions of the pocket are shown in Figure 9(d). This procedure may be useful for creating scleral pockets for implants to treat presbyopia.

\subsection{Subsurface Volume Pattern Photodisruption}

To evaluate the completely enclosed photodisruption pattern, a rectangular prism geometry was scanned beneath the surface as shown in Figure 10(b) using the $775 \mathrm{~nm}$ laser. The spot separation in this pattern was $3 \mu \mathrm{m}$ in every dimension. The rectangular prism was $100 \mu \mathrm{m}$ wide, $100 \mu \mathrm{m}$ deep, and $3 \mathrm{~mm}$ long. Subsequently, the sample was cut perpendicular to the tissue surface to expose a $100 \times 100 \mu \mathrm{m}$ square cross section. The incision as shown in Figure 10(a) shows two significant 


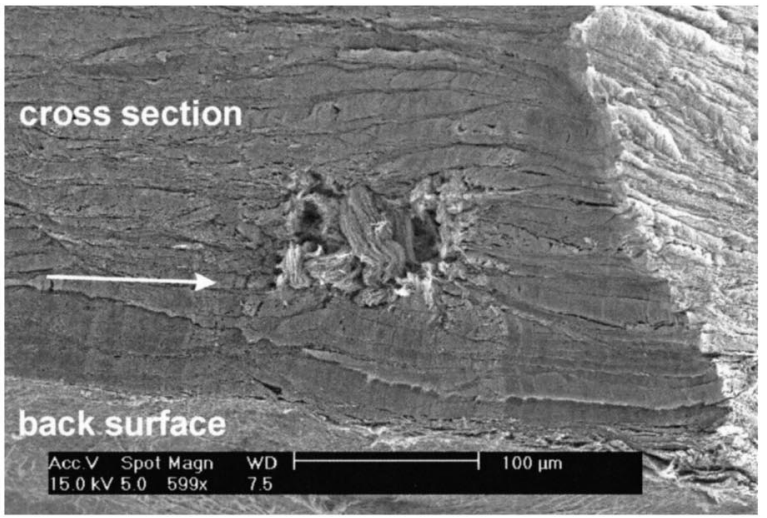

(a)



(b)

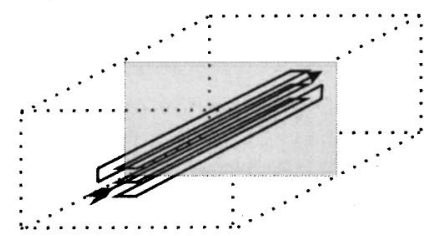

(c)

Fig. 10 Subsurface vessel in sclera. (a) SEM image of vessel. Note the small size of the vessel and that there is little collateral damage in the incision. The debris in the incision appears to be fibrous. (Laser parameters: $2.6 \mu$ J, $150 \mathrm{fs}, 775 \mathrm{~nm}, 1 \mathrm{kHz}, 1.5 \mu \mathrm{m}$ FWHM spot, $3 \mu \mathrm{m}$ spot separation.) (b) Potential application of creating a subsurface vessel for fluid conduction analogous to Schlemm's Canal. (c) Raster pattern at laser focus.

features. First, the tissue has not been altered above and below the incision. Second, there is debris in the treated volume that maintains some lamellar structure of the sclera. Irregularities in the shape of the photodisrupted area may be due to a variety of reasons, including scattering of the laser light in the tissue, laser beam stability (energy and position), and processing distortion.

In order to compare the quality of incisions between 775 and $1060 \mathrm{~nm}$ lasers, the $1060 \mathrm{~nm}$ laser was used to cut a similar geometry. Figure 11 shows a $50 \times 50 \mu \mathrm{m}$ cross section of a rectangular prism cut with a $5 \mu \mathrm{m}$ spot separation using the $1060 \mathrm{~nm}$ laser. In this instance, there is no damage above and below the incision, but the debris in the incision has a different structure of that shown in Figure 10(c) using the $775 \mathrm{~nm}$ laser. When viewed at higher magnification in Figure 12, a sharp boundary between the photodisruption debris and the adjacent tissue is seen. The debris from the 1060 nm laser ablations is quite disorganized, as compared with the more regular structure seen with the $775 \mathrm{~nm}$ ablations shown in Figure 10(c). Adjacent to this area is a $2-10 \mu \mathrm{m}$ wide region that contains undisrupted, but debris-coated fibers, which were not present in $775 \mathrm{~nm}$ ablations.

\section{Discussion}

We have demonstrated in an in vitro model that human sclera can be made surgically accessible for high precision intrascleral photodisruption if the sclera's natural light scattering properties are reduced by dehydration. Similar to observations in the cornea, minimal collateral damage was observed in the surrounding structures adjacent to the photodisrupted volume. ${ }^{21}$ A variety of incisional geometries can be created as long as the pattern is formed in a deep to superficial manner, so that cavitation bubbles produced during photodisruption do


Fig. 11 Cross section of lesion created in sclera. (a) Hyper intense region denotes $50 \times 50 \mu \mathrm{m}$ rectangular cross section of lesion created in sclera. The tissue above and below the lesion appear intact. The thick line denotes the top surface of the tissue. (b) Intact collagen fibers. The periodic banding structure of collagen is visible. (Laser parameters: $5 \mu$ J, $500 \mathrm{fs}, 1060 \mathrm{~nm}, 1 \mathrm{kHz}, 5 \mu \mathrm{m}$ FWHM spot, $5 \mu \mathrm{m}$ spot separation.) 
High Precision Subsurface Photodisruption ...

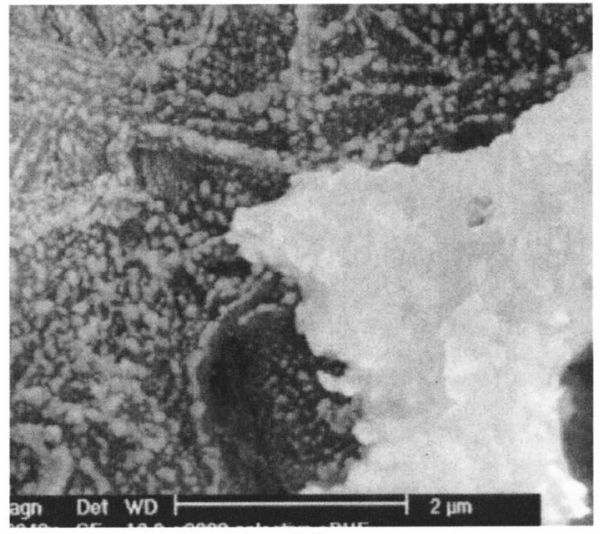

(a)

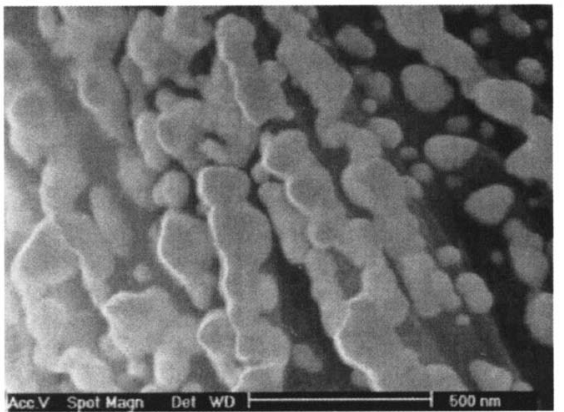

(b)

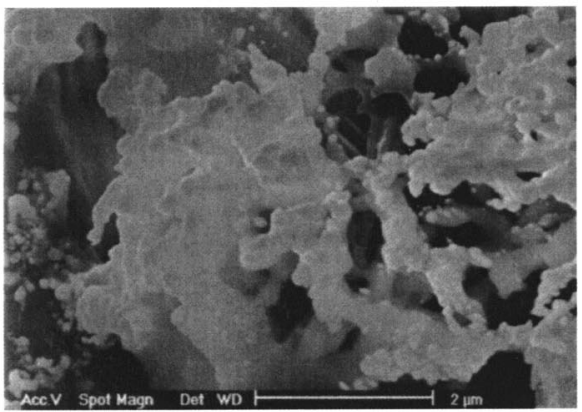

(c)

Fig. 12 Higher magnification of lesion zone. (a) A boundary between solid debris and debris-coated fibers. (b) Debris seems to be coating the fibers adjacent to the debris region. (c) The debris is disorganized collagen bundle.

not interfere with the focusing of the laser beam. Partial and full-thickness transcleral channels, subsurface pockets, and subsurface volumetric photodisruption are examples of possible geometries. In patterns where surface openings are not created (or are inadequate for the volume of debris) the photodisrupted material remains inside the tissue, appearing bright in the electron micrographs. Although the mechanism accounting for the debris coating adjacent collagen fibers is not known, ejection of a portion of the debris into the surrounding tissue seems likely.

A number of additional studies is required to determine if and how ultrashort laser pulses can be used for actual surgical procedures in the sclera. First, in vivo studies, must determine if enough optical clearing occur after subconjunctival injection of Hypaque to allow low energy photodisruption with femtosecond pulses. Second, physiologic effectiveness must be evaluated. For example, in glaucoma procedures, residual tissue debris may block aqueous flow, although hydraulic conductivity may be altered enough in the treated volume to increase fluid outflow. In this case, the photodisrupted debris could help to keep laser created transscleral channels open. Finally, in vivo testing is required to evaluate effects evaluate effects that may not be apparent using this in vitro techniques, such as toxicity from the dehydrating agent or wound healing after laser ablation. ${ }^{22}$ In addition, similar techniques for subsurface photodisruption may be possible in other tissues, such as the skin, where similar optical clearing effects are possible. $^{23}$
The differences seen between the $1060 \mathrm{~nm} 500$ fs laser and the $775 \mathrm{~nm} 150 \mathrm{fs}$ laser are interesting, although many other parameters also differed in the two systems, including wavelength, spot size, pulse duration, and scanning density. More detailed investigations are necessary to characterize the temporal and spatial profile of the pulses applied, since the standard FWHM measurement of the temporal duration may not be adequate to determine the breakdown characteristics of the pulse. ${ }^{24}$ Regardless, both laser sources provide a method of subsurface photodisruption in human sclera, which have the potential to be used in a clinical procedure.

\section{Acknowledgments}

The authors thank Professor Valery V. Tuchin for technical discussions regarding induced transparency in sclera, the Michigan Eye-Bank for providing tissue, and Bruce Donohoe of the University of the Michigan Cell Biology Lab for mounting tissue. The work was done with the support of Dr. John F. Mansfield and Dr. Corinna J. Wauchope of the University of Michigan North Campus Electron Microbeam Analysis Laboratory. This research was sponsored by the National Science Foundation (through STC PHY 8920108, University of Michigan, and SBIR 99-01726, IntraLase Corporation). 


\section{References}

1. X. Liu, D. Du, and G. Mourou, "Laser ablation and micromachine with ultrashort laser pulses," IEEE J. Quantum Electron. 33(10), 1706-1716 (1997).

2. T. Juhasz, F. H. Loesel, R. M. Kurtz, C. Horvath, J. F. Bille, and G. A. Mourou, "Corneal refractive surgery with femtosecond lasers," IEEE J. Sel. Top. Quantum Electron. 15(4), 902-910 (1999); G. Maatz, A. Heisterkamp, H. Lubatschowski, S. Barcikowski, C. Fallnich, H. Welling, and W. Ertmer, "Chemical and physical side effects at application of ultrashort laser pulses for intrastromal refractive surgery," J. Opt. A: Pure Appl. Opt. 2, 59-64 (2000).

3. W. Kautek and J. Kruger, "Femtosecond pulse laser ablation of metallic, semiconducting, ceramic, and biological materials," Proc. SPIE 2207, 600-611 (1996).

4. A. Vogel and J. Noack, "Shock wave energy and acoustic energy dissipation after laser induced breakdown," Proc. SPIE 3254, 180189 (1998).

5. E. N. Glezer and E. Mazur, "Ultrafast-laser driven micro-explosions in transparent materials," Appl. Phys. Lett. 71(7), 882-884 (1998).

6. Z. S. Sacks, R. M. Kurtz, R. Fenn, F. Loesel, and T. Juhasz, "Laser spot size as a function of tissue depth and laser wavelength in human sclera," Proc. SPIE 3255(12), 67-76 (1998).

7. HYPAQUE 76, diatrizoate meglumin 76\%, Nycomed Inc., Princeton, $\mathrm{NJ}$

8. V. V. Tuchin, I. L. Maksimova, D. A. Zimnyakov, I. L. Kon, A. H. Mavlutov, and A. A. Mishin, "Light propagation in tissues with controlled optical properties," J. Biomed. Opt. 2(4), 401-417 (1997).

9. D. A. Zimnyakov, V. V. Tuchin, A. A. Mishin, I. L. Kon, and A. N. Serov, "In vitro human sclera structure analysis using tissue optical immersion effect," Proc. SPIE 2673(44), 233-242 (1996).

10. S. Vaezy and J. I. Clark. "A quantative analysis of transparency in human sclera and cornea using Fourier methods," J. Microsc. 163(1), 85-94 (1991); S. Vaezy and J. I. Clark, "Quantitative analysis of the microstructure of human cornea and sclera using 2-D Fourier methods," ibid. 175(2), 93-99 (1994).

11. This procedure was provided by the Kellogg Eye Bank for preserving sclera for transplantation.
12. P. O. Rol, "Optics for transscleral laser applications," Ph.D dissertation, Swiss Federal Institute of Technology, Zurich, Switzerland, 1991, ETH No. 9665.

13. Z. S. Sacks, "Femtosecond transscleral photodisruption for the treatment of glaucoma," PhD dissertation, University of Michigan, 2000.

14. C. Horvath, A. Braun, H. Liu, T. Juhasz, and G. A. Mourou, "Compact directly diode-pumped femtosecond Nd:glass chirped-pulseamplification laser system," Opt. Lett. 22(23), 1790-1792 (1997).

15. A. Vogel, K. Nahen, D. Theisen, R. Bringruber, R. J. Thomas, and B. A. Rockwell, "Influence of optical aberrations on laser-induced plasma formation in water and their consequences for intraocular photodisruption," Appl. Opt. 38(16), 3636-3643 (1999).

16. M. Born and E. Wolf, Principles of Optics, 6th ed., Cambridge University Press, Cambridge (1997).

17. N. J. Fullwood and K. M. Meek, "A synchrotron X-ray study of the changes occurring in the corneal stroma during processing for electron microscopy," J. Microsc. 169(1), 53-60 (1993).

18. A. S. Craig, J. B. Robertson, and D. A. D. Parry, "Preservation of corneal collagen fibril structure using low-temperature procedures for electron microscopy," J. Ultrastruct. Res. 96, 172-175 (1986).

19. M. B. Shields, Glaucoma, 4th ed., Williams \& Wilkins, Baltimore (1998).

20. Treatment of Presbyopia and Other Eye Disorders, US Patent No. $5,354,331$

21. D. Stern, R. W. Schoenlein, C. A. Puliafito, E. R. Dobi, R. Birngruber, and J. G. Fujimoto, "Corneal ablation by nanosecond, picosecond, and femtosecond lasers at 532 and $625 \mathrm{~nm}$," Arch. Ophthalmol. (Chicago) 107, 587-592 (1989).

22. A. C. Tien, S. Backus, H. Kapteyn, M. Murnane, and G. A. Mourou, "Short-pulse laser damage in transparent materials as a function of pulse duration,” Phys. Rev. Lett. 82(19), 3883-3886 (1999).

23. G. Vargas, E. K. Chan, J. K. Barton, H. G. Rylander III, and A. J. Welch. "Use of an agent to reduce scattering in skin," Lasers Surg. Med. 24, 133-141 (1999).

24. A. J. Welch and M. J. C. van Gemert, Optical-Thermal Response of Laser-Irradiated Tissue, Plenum, New York (1995). 